\title{
Organism Part Location
}

National Cancer Institute

\section{Source}

National Cancer Institute. Organism Part Location. NCI Thesaurus. Code C103200.

Anatomical placement of a particular portion of an organism. 